\title{
Optimizing Terminal Delivery of Perishable Products considering Customer Satisfaction
}

\author{
Xuping Wang, ${ }^{1,2}$ Xiaoyu Sun, ${ }^{1}$ Jie Dong, ${ }^{2}$ Meng Wang, ${ }^{2}$ and Junhu Ruan ${ }^{1,3,4}$ \\ ${ }^{1}$ Institute of Systems Engineering, Dalian University of Technology, No. 2, Linggong Road, Dalian 116024, China \\ ${ }^{2}$ School of Business, Dalian University of Technology, No. 2, Dagong Road, Panjin 124221, China \\ ${ }^{3}$ College of Economic and Management, Northwest A\& $F$ University, Yangling 712100, China \\ ${ }^{4}$ Department of Industrial and Systems Engineering, The Hong Kong Polytechnic University, Hung Hom, Hong Kong
}

Correspondence should be addressed to Xuping Wang; wxp@dlut.edu.cn

Received 28 July 2016; Revised 20 November 2016; Accepted 14 December 2016; Published 15 February 2017

Academic Editor: Rita Gamberini

Copyright (c) 2017 Xuping Wang et al. This is an open access article distributed under the Creative Commons Attribution License, which permits unrestricted use, distribution, and reproduction in any medium, provided the original work is properly cited.

\begin{abstract}
Freshness of products and timeliness of delivery are two critical factors which have impact on customer satisfaction in terminal delivery of perishable products. This paper investigates how to make a cost-saving vehicle scheduling for perishable products by maximizing customer satisfaction. Customer satisfaction is defined from the two aspects of freshness and time window. Then we develop a priority function based on customer satisfaction and use the hierarchical clustering method to identify customer service priority. Based on the priority, a multiobjective vehicle scheduling optimization model for perishable products is formulated to maximize customer satisfaction and minimize total delivery costs. To solve the proposed model, a priority-based genetic algorithm (PB-GA) is designed. Numerical experiments and sensitivity analysis are performed to show the validity and advantage of our approach. Results indicate that PB-GA can achieve better solutions than traditional genetic algorithm. The improvement of customer satisfaction is higher than the decrease rate of total costs within a certain shelf life range, which reveals that the proposed method is applicable to the terminal delivery of perishable products.
\end{abstract}

\section{Introduction}

With the popularization of online shopping and the improvement of delivery service, more and more customers buy perishable products on Business-to-Customer (B2C) platforms. However, freshness of products and timeliness of delivery are two critical factors affecting customer satisfaction in B2C experience [1]. Thus, it is an important issue to make reasonable delivery plans for perishable products with the consideration of customer satisfaction in terminal delivery.

Perishable products, such as fruits, vegetables, and meat, have short delivery timespans. These products may start deteriorating from the moment they are produced and the freshness appears to be decreasing as transportation time elapses until spoilt $[2,3]$. Freshness is one of the primary concerns when customers buy perishable products. However, in China, the damage rate of perishable products reaches up to $30 \%$, much higher than the $5 \%$ in developed countries.
Especially for terminal delivery, it makes great influences on customer's overall satisfaction. Therefore, distributors require to take into account the freshness factor in terminal delivery planning. Moreover, timeliness is another important factor of customer satisfaction. If distributors are not able to deliver products on time, the customer satisfaction probably decreases.

Taking these two factors into consideration, it is a difficulty for logistics service providers to ensure freshness and timeliness during terminal delivery because perishable products need to be handled in a special way not the traditional cost-saving way. From the customer satisfaction view, we study a multiobjective vehicle scheduling problem for perishable products in terminal delivery.

To sum up, our contribution includes three aspects. (1) We define a customer priority function considering the freshness and time window to qualify customer satisfaction and use the hierarchical clustering method to classify customers 
into different service priorities. (2) Taking customer service priority as one of constraints, we establish a multiobjective vehicle scheduling optimization model for perishable products. The objective functions consist of maximizing customer satisfaction and minimizing total costs. (3) Then we design a priority-based genetic algorithm for the proposed model, which could produce satisfactory terminal delivery plans.

The remainder of this paper is organized as follows. Section 2 presents a brief review on related studies. In Section 3, a mathematical model of multiobjective vehicle scheduling optimization for perishable products is formulated. To solve the model, a priority-based genetic algorithm is designed in Section 4. In Section 5, numerical experiments and sensitivity analysis are presented to show the validity and advantage of our work. The paper is concluded in Section 6.

\section{Literature Review}

Considering customer satisfaction in perishable products delivery has been continuously a concern in both academic research and industry application. In this review, we focus on two parts directly related to delivery problems for perishable products. Firstly, we look into what has been done in perishable products distribution with time windows and the definition of customer satisfaction. Secondly, some related studies on the multiobjective modelling for VRP are briefly reviewed.

2.1. VRPTW for Perishable Products and Definition of Customer Satisfaction. The well-known vehicle routing problem with time windows (VRPTW) has been discussed deeply in the literature. In recent years, many scholars have studied VRPTW for perishable products in various aspects. Osvald and Stirn [4] extended a heuristic algorithm for distributing fresh vegetables where perishability was set as a critical factor. The problem was formulated as a VRPTW with time-dependent travel time (VRPTWTD). Considering the randomness of perishable products in delivery process, Hsu et al. [5] extended the VRPTWTD model that the perishability cost was served as a stochastic manner. Chen et al. [6] focused on production scheduling and vehicle routing with time windows to maximize the expected total profit of supplier. Ahumada and Villalobos [7] and Yan et al. [8] followed a produce through all stages of production and distribution to build an integrated model for perishable products. Wang and Yu [9] took different delivery modes into consideration and established a perishable product delivery network to minimize the total costs. Coelho and Laporte [10] compared two suboptimal policies and computed optimal joint replenishment and delivery decisions for perishable products effectively. Firoozi et al. [11] developed an efficient network for storage and perishable products distribution. Ruan and Shi [12] formulated an Internet of Things-based framework for monitoring and assessing the freshness of intransit fruits. These studies mainly belonged to traditional VRPTW, and most researches took the minimization of total costs as the optimization objective. They converted the damage rate of perishable products into corresponding damage cost and entrusted with different weights for each cost. However, the weighting method has a certain degree of subjectivity. Moreover, perishable products have the characteristic of perishability and the spoilt products can decrease customer satisfaction tremendously. In this sense, the above studies only simply considered the economic perspective and ignored customer satisfaction for distributing perishable products.

Some researchers took customer satisfaction into consideration. Rong et al. [13] determined product deterioration by time and temperature. If the customer requirement was not satisfied, a penalty cost would be incurred for the spoilt food. Considering quality time window, Jia et al. [14] formulated a production-distribution-inventory model to control product quality and satisfy customer requirement. Amorim and Almada-Lobo [15] examined relationship between distribution scenarios and the cost-freshness trade-off. Cao et al. [16] introduced fuzzy appointment time to reflect customer preference time window and defined service start time of fuzzy membership functions as customer satisfaction. The above studies play an important role for the following research works, but the studies mainly describe customer satisfaction from the view of delivery timeliness. For perishable products, freshness is a key factor affecting customer satisfaction while these studies do not take into account the perishability.

2.2. Multiobjective Modelling for VRP. Multiobjective modelling for VRP is commonly used in fields of microcalamities management and emergency logistics, which take humanitarian factors into consideration [17-22]. With perishability concerned in this paper, we mainly concentrate on multiobjective VRP for perishable products. Amorim et al. [23] developed a novel model that decoupled the minimization of delivery costs from the maximization of the freshness state of delivered products. Atashbar and Baboli [24] designed a multiobjective routing problem for perishable items in a disaster relief. In this situation, the first objective was to maximize satisfaction and demands coverage, and the second objective was to minimize the total time needed to transfer perishable products. Bortolini et al. [25] constructed a multiobjective perishable distribution model considering distribution costs, delivery time, and carbon emissions. Rahimi et al. [26] applied social issue to perishable products delivery, aiming at minimizing distribution costs and maximizing social issue which was calculated by vehicle accident rate and number of expired products. Wang et al. [27] proposed an effective distribution route that minimized the total costs and maximized freshness state of delivered products. We noted that the above studies enhanced the traditional multiobjective models for VRP to make them more adaptable for perishable products delivery. However, these studies formulated multiobjective models from different perspectives which made objective functions different from each other. In this paper, our focus is mainly on maximizing customer satisfaction and minimizing total logistics costs.

According to the above analysis, we try to define customer satisfaction from the aspects of freshness and time window. Moreover, we combine customer satisfaction with terminal delivery which can make a reasonable vehicle scheduling for 
perishable products. A multiobjective mathematical model and hybrid algorithm are proposed to solve the problem.

\section{Problem Description and Formulation}

3.1. Problem Description. The delivery processes of perishable products are as follows: customers buy perishable products on B2C platform and submit orders to suppliers. When vehicles start their travel from suppliers, the freshness is assumed to be perfect. During long-distance transportation to local distribution centers (LDCs), the freshness decreases based on freshness reduction rate of each product. In this paper, we deal with a vehicle routing problem from a LDC (depot) to customers for delivering perishable products.

A terminal delivery network is described by a graph $G(V, E)$, where $V$ is the set of nodes and $E$ is the set of arcs. In the network, the node set $V$ consists of the set of customers $N$ and the depot is denoted as 0 , and the arc set $E$ represents shortest distance routes among nodes in the network. The assumptions are as follows:

(1) Demand of each customer is less than the maximum capacity of vehicles.

(2) A type of perishable products is with the same shelf life.

(3) A type of vehicles is with the same capacity.

3.2. Definitions of Customer Satisfaction and Service Priority. As discussed in the Introduction, customer satisfaction mainly comes from the freshness and timeliness of delivered products. In this section, we define customer satisfaction from these two factors, respectively, and then convert it into customer service priority.

(1) Satisfaction on Freshness. In general, freshness of perishable products often decreases with the duration time of transportation. According to the existing studies, freshness of unit product purchased by customer $i$ can be defined as $\theta_{i}=2-e^{(\ln 2 / T) t_{0 i}}$, where $t_{0 i}$ is the transportation time from depot to customer $i$ and $T$ is the shelf life of products [28]. Additionally, the more the customer demands are, the greater the loss will be for the same duration time. Therefore, we take the proportion of total demands into consideration, so the freshness satisfaction for customer $i$ is defined as

$$
\frac{\theta_{i} \cdot q_{i}}{\sum_{i \in N} q_{i}}
$$

where $q_{i}$ is the demand of customer $i$ and $N$ is the number of customers.

(2) Satisfaction on Time Window. Delivery plans often route vehicles based on customer specific time window. But in many real-life cases, the deviation of time window does not directly incur any penalty cost, and it only has negative effect on satisfaction level [29]. Hence, we apply the fuzzy theory

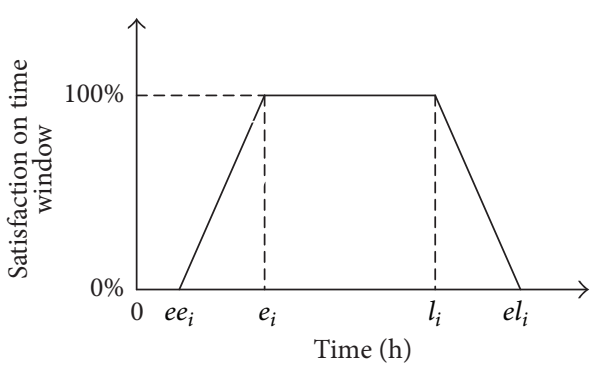

FIGURE 1: Satisfaction on time window.

to describe this reality. The satisfaction on time window for customer $i$ is defined as

$$
U\left(t_{i}\right)= \begin{cases}\frac{\left(t_{i}-e e_{i}\right)}{\left(e_{i}-e e_{i}\right)}, & t_{i} \in\left[e e_{i}, e_{i}\right) \\ 100 \%, & t_{i} \in\left[e_{i}, l_{i}\right] \\ \frac{\left(e l_{i}-t_{i}\right)}{\left(e l_{i}-l_{i}\right)}, & t_{i} \in\left(l_{i}, e l_{i}\right] \\ 0, & t_{i} \notin\left[e e_{i}, e l_{i}\right],\end{cases}
$$

where $\left[e_{i}, l_{i}\right]$ represents the expected arrival time interval for customer $i$ during which the satisfaction level will be $100 \%$. The lower bound $e e_{i}$ represents the earliest arrival time that a customer can endure when a service starts earlier than $e_{i}$. Similarly, the upper bound $e l_{i}$ represents the latest arrival time that customer can endure when service starts later than $l_{i}$. When customer is served within $\left[e e_{i}, e_{i}\right]$ or $\left[l_{i}, e l_{i}\right]$, the satisfaction level is with an ascending or a descending linear relationship with actual arrival time $t_{i}$, respectively. Otherwise, the satisfaction level will be 0 . Figure 1 denotes the satisfaction on time window at different arrival times.

(3) Customer Satisfaction. Both freshness and time window have great influence on customer satisfaction. Based on the definitions in Formulas (1) and (2), the customer satisfaction can be expressed as

$$
\frac{\alpha \sum_{i \in N}\left(\theta_{i} \cdot q_{i}\right)}{\sum_{i \in N} q_{i}}+\frac{\beta \sum_{i \in N} U\left(t_{i}\right)}{N}
$$

where $\alpha$ is freshness sensitivity coefficient, $\beta$ is time window sensitivity coefficient, and $\alpha+\beta=1$.

(4) Service Priority Based on Customer Satisfaction. The priority refers to the service order for each customer. Higher priority often needs earlier service. In this paper, we identify service priority based on the customer satisfaction on both freshness and time window. Under constraints of the number of vehicles and capacity limits, it is reasonable to prioritize the customers in order to maximize the freshness satisfaction and meet expected time window. Based on the above analysis 
on customer satisfaction, we construct a priority evaluation function for customer $i$ as follows:

$$
\begin{aligned}
P(i)= & \alpha\left(1-\theta_{i}\right) \frac{q_{\max }-q_{i}}{q_{\max }} \\
& +\beta\left(\frac{\left|t_{0 i}-e_{i}\right|}{\left|l_{i}-e_{i}\right|}+\frac{\left|l_{i}-t_{0 i}\right|}{\left|l_{i}-e_{i}\right|}\right),
\end{aligned}
$$

where the freshness of perishable products for customer $i$ is $\theta_{i}=2-e^{\ln 2\left(\left(d_{o i} / v\right) / T\right)}, q_{\max }$ is the maximal demand of all customers, and $t_{0 i}$ is the travel time from depot to customer $i$.

The first item in Formula (4) reflects the freshness factor. Obviously, the smaller the distance $d_{0 i}$ and the bigger the demand $q_{i}$, the smaller the first item, that is, to provide high service priorities for the customers with closer distances to depot and larger demands. Similarly, the second item reflects the time window factor. When $t_{0 i} \in\left[a_{i}, b_{i}\right]$, then $\mid t_{0 i}-$ $e_{i}|/| l_{i}-e_{i}|+| l_{i}-t_{0 i}|/| l_{i}-e_{i} \mid=1$; when $t_{0 i} \notin\left[a_{i}, b_{i}\right]$, then $\left|t_{0 i}-e_{i}\right| /\left|l_{i}-e_{i}\right|+\left|l_{i}-t_{0 i}\right| /\left|l_{i}-e_{i}\right|>1$, that is, to provide high service priorities for the customers who are more consistent with the expected time window. Thus, the smaller value of $P(i)$, the higher service priority for customer $i$.

Meanwhile, we deal with Formula (4) to eliminate dimension. Let $U_{1}(i)$ be the first item in Formula (4), and $U_{1} \max$ is the maximum of $U_{1}(i)$. Similarly, let $U_{2}(i)$ be the second item, and $U_{2}$ max is the maximum of $U_{2}(i)$. Thus, standardized priority evaluation function for customer $i$ is defined:

$$
P^{\prime}(i)=\alpha \frac{U_{1}(i)-U_{1} \min }{U_{1} \max -U_{1} \min }+\beta \frac{U_{2}(i)-U_{2} \min }{U_{2} \max -U_{2} \min } .
$$

Formula (5) means that when $\alpha=0, \beta \neq 0$, the evaluation function only considers the time window factor and does not take freshness into consideration. On the contrary, when $\alpha \neq 0, \beta=0$, it only considers the freshness factor and ignores the time window. Similar to $P(i)$, the smaller the value of $P^{\prime}(i)$, the higher the service priority for customer $i$. At the same time, all customers will be served from high priority to low.

(5) Hierarchical Clustering for Service Priority. According to the above analysis, we can calculate $P^{\prime}(i)$ for each customer, but different values of $P^{\prime}(i)$ refer to different priority levels. Moreover, too many levels may greatly limit the selection of delivery routes. Thus, it is better to assign customers with similar values of $P^{\prime}(i)$ into the same priority level, which can also mean the same cluster. Hierarchical clustering [30] is a useful pattern recognition method for organizing items into groups or clusters. The degree of similarity is high between items in the same cluster and low in different clusters. Given a set of $N$ items to be clustered, the basic procedures of the hierarchical clustering are as follows.

Step 1. Assign $N$ items to $N$ clusters. Each cluster contains just one item. Calculate the $N * N$ distance (or similarity) matrix.

Step 2. Merge the two clusters with the largest similarity into one single cluster. In this step, two clusters are reduced and one new cluster is created.
Step 3. Compute distances (or similarities) between the new cluster and each of the old clusters. Update distance matrix.

Step 4. Repeat Steps 2 and 3 until all items are clustered into a single cluster of size $N$.

Based on the hierarchical clustering, we can group customer service priorities into clusters. According to results of clustering, we redefine customer service priority as $P_{i}$ for customer $i$ (each value of $P_{i}$ represents a cluster). More details are stated in Section 5.2.

3.3. Optimization Model. In this study, we deal with terminal delivery of perishable products considering customer satisfaction using a vehicle scheduling model. The parameters and variables are defined as follows.

\section{Parameters}

$N$ : a set of customers, $N=\{n|n=1,2, \ldots| N \mid$,$\} .$

$N^{+}$: a set of depot and customers where $n=0$ denotes the depot, $N^{+}=\{n|n=0,1, \ldots| N \mid$,$\} .$

$K$ : a set of vehicles, $K=\{k|k=1,2, \ldots| K \mid$,$\} .$

$c_{0}$ : transportation cost per unit distance.

$d_{i j}$ : distance between customers $i$ and $j, i, j \in N^{+}$.

$F_{c}$ : fixed vehicle cost per trip.

$\mathrm{ST}_{i}$ : service time for customer $i(\mathrm{~min})$.

$\Delta t$ : long-distance travel time before arriving LDC (h).

Q: maximum capacity per vehicle $(\mathrm{kg})$.

$q_{i}$ : demand of customer $i(\mathrm{~kg})$.

$v_{0}$ : average travel speed $(\mathrm{km} / \mathrm{h})$.

$T$ : shelf life of perishable product (h).

\section{Variables}

$\theta_{i}$ : freshness of products for customer $i, i \in N$.

$t_{i j}$ : travel time from customer $i$ to $j, i, j \in N^{+}$.

$t_{i}$ : arrival time of customer $i, t_{0}=0$ is the initial time.

$\left[a_{i}, b_{i}\right]$ : delivery time window of customer $i$.

$P_{i}$ : priority of customer $i$.

$x_{i j k}$ : binary variable, $x_{i j k}=1$ if vehicle $k$ travels from customer $i$ to $j$; otherwise $x_{i j k}=0$.

On the basis of Section 3.2, we can determine the priority of each customer. Then a mathematical model of multiobjective vehicle scheduling optimization for perishable 
products considering customer satisfaction can be given as follows:

$$
\text { Maximize } \quad \mathrm{LP} 1=\frac{\alpha \sum_{i \in N}\left(\theta_{i} \cdot q_{i}\right)}{\sum_{i \in N} q_{i}}+\frac{\beta \sum_{i \in N} U\left(t_{i}\right)}{N}
$$

Minimize LP2

$$
=c_{0} \sum_{i \in N^{+}} \sum_{j \in N^{+}} \sum_{k \in K} d_{i j} x_{i j k}+F_{c} \sum_{j \in N} \sum_{k \in K} x_{0 j k}
$$

Subject to: $\quad \sum_{j \in N} \sum_{k \in K} x_{0 j k} \leq K$

$\sum_{j \in N} x_{0 j k}=\sum_{j \in N} x_{j 0 k} \leq 1, \quad \forall k \in K$

$\sum_{i \in N^{+}} \sum_{j \in N^{+}} x_{i j k} q_{i} \leq Q, \quad \forall k \in K$

$\sum_{i \in N^{+}} \sum_{k \in K} x_{i j k}=1, \quad \forall j \in N$

$\sum_{i \in N^{+}} \sum_{k \in K} x_{j i k}=1, \quad \forall j \in N$

$\left(P_{j}-P_{i}\right) x_{i j k} \geq 0$

$$
\begin{gathered}
\forall i, j \in N^{+}, i \neq j, k \in K \\
\theta_{i}=2-e^{\ln 2\left(\left(t_{i}+\Delta t\right) / T\right)}, \quad \forall i \in N^{+} \\
t_{j}=\left(t_{i}+\frac{d_{i j}}{v_{0}}+\mathrm{ST}_{i}\right) x_{i j k}, \\
\forall i, j \in N^{+}, k \in K \\
x_{i j k} \in\{0,1\}, \quad i, j \in N^{+}, k \in K .
\end{gathered}
$$
Then we transform constraints into fitness function and evaluate it based on sorting matrix; in addition, improved genetic operators are used to ensure customer service priority in crossover and mutation operations. The procedure of PB-GA is shown in Figure 2. The details will be stated below.

4.1. Encoding and Initial Population. We use sequence encoding with natural numbers $0 \sim n$ to express delivery routes, where $n=0$ means the depot. Feasible solutions for initial population are created by two strategies: the first strategy is "Priority Rule + Nearest Neighbor Rule" with probability $\rho$, and the second one is random generation with probability $(1-\rho)$. At first we define two rules.

Rule 1 (priority rule). In each subroute, delivery order of each vehicle is carried out by customer service priority from high to low.

Rule 2 (nearest neighbor rule). Select the nearest Euclidean distance from current node as the next delivery node.

On the basis of these two rules, the "Priority Rule + Nearest Neighbor Rule" strategy is explained in detail as in the following steps.

Step 1. Sort customer service priority $P_{i}$ from high to low. If there is more than one customer with the same value of $P_{i}$, then sort them according to the ascending order in Euclidean distance from the LDC.

Step 2. Select the first customer point from sort order in Step 1 ; if this customer is not served by any vehicle, then set it as Seed which is the first service point for a vehicle and then move to Step 3. If all customers have been served, then move to Step 6.

Step 3. Select other customer points to generate the route. Pick the next unserved customer point based on Rules 1 and 2 and calculate total demands $\sum q_{i}$ in the subroute. If $\sum q_{i} \leq Q$, then combine it to form the subroute and move to Step 5; otherwise move to Step 4.

Step 4. Iterate Step 3, until the total amount of current subroute exceeds the maximum capacity of vehicle. Then move to Step 5.

Step 5. Stop the current subroute, and move back to Step 2.

Step 6. Output vehicle routing results. 

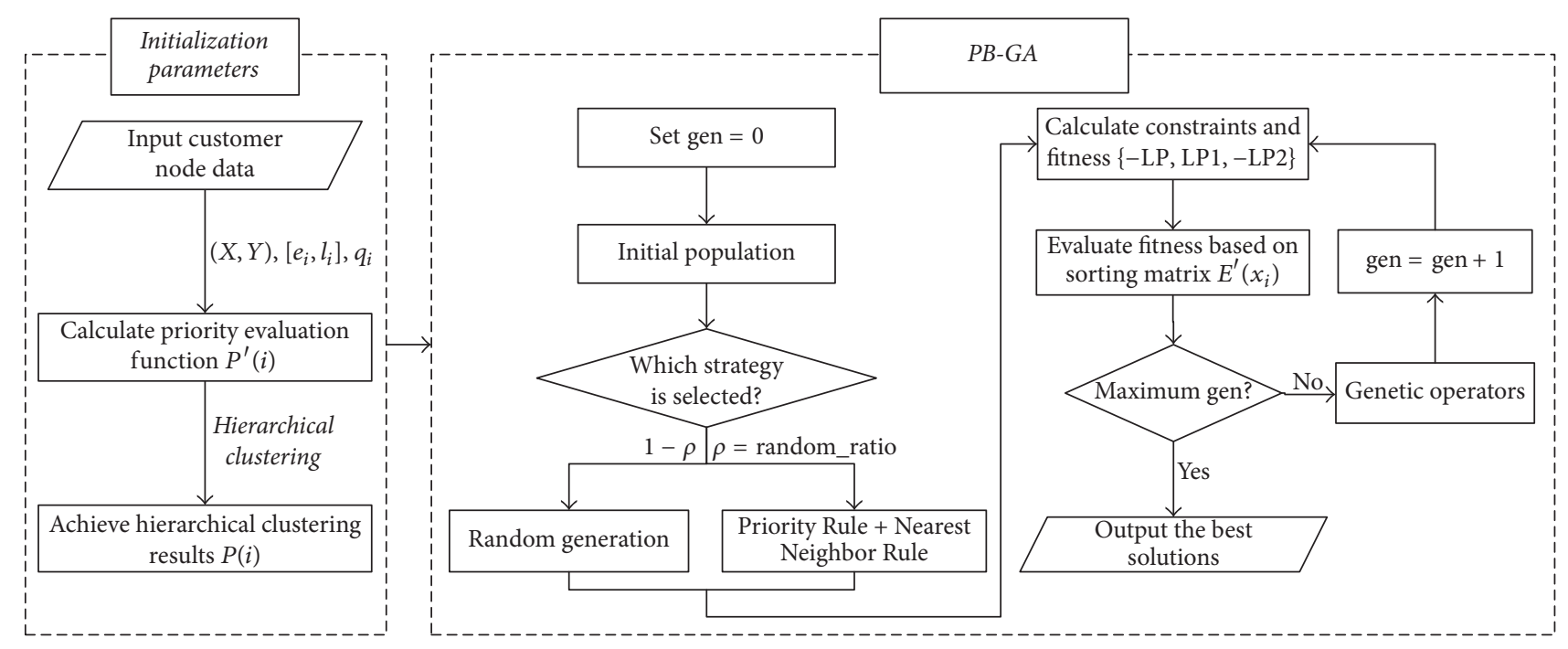

Figure 2: The procedure of PB-GA.

\subsection{Genetic Operators}

4.2.1. Selection Operator. A roulette wheel selection mechanism will be used to select the prospective parents based on their fitness computed by the evaluation function. The probability of an individual being selected is positive proportional to fitness. That is, the probability of selected individual $i$ is $p_{i}=F_{i} / \sum_{i \in N} F_{i}, i=1,2, \ldots, N$, where $N$ is the population size and $F_{i}$ is the fitness of individual $i$.

4.2.2. Crossover and Mutation Operators. Classical Order Crossover (OX) can better retain neighbor relationship in chromosome. But it may also produce infeasible route sequence because of priority violation. Therefore, this paper employs a priority-based crossover. When the initial solution is generated by "Priority Rule + Nearest Neighbor Rule," crossover operator should not be applied to the Seed (the first delivery point of a subroute). Hence, it can reduce the change of order priority in a route. The mutation operator first selects randomly two break points in a chromosome and then makes an exchange of these two points. Also the mutation operator should not be applied to the Seed.

4.3. Constraints Handling and Fitness Function. To meet the constraints of the number of vehicles, capacity of vehicle, and service priority, this paper converts these three constraints into a penalty term LP and adds it as a new objective function. The expression of LP is given by

$$
\begin{aligned}
\mathrm{LP}= & M_{1} \max \left(\sum_{j \in N} \sum_{k \in K} x_{0 j k}-K, 0\right) \\
& +M_{2} \max \left(\sum_{i, j \in N^{+}} \sum_{k \in K} x_{i j k} q_{i}-Q, 0\right)
\end{aligned}
$$

$$
+M_{3} \max \left(\sum_{i, j \in N^{+}} \sum_{k \in K}\left(P_{i}-P_{j}\right) x_{i j k}, 0\right) \text {, }
$$

where $M_{1}, M_{2}$, and $M_{3}$ (large positive numbers) represent penalty coefficients of the number of vehicles, capacity of vehicle, and service priority, respectively. Constraints handling and evaluation of fitness function are as follows.

Step 1. LP represents constraints of this model, so we set LP as the first goal. Subsequently, the other objectives are downgraded in turn. Thus, three objectives are reformed for each individual: $\max -\mathrm{LP}, \max \mathrm{LP} 1$, and $\max -\mathrm{LP} 2$. And we express the multiple objectives as $\mathrm{Obj}_{1}=-\mathrm{LP}, \mathrm{Obj}_{2}=\mathrm{LP} 1$, and $\mathrm{Obj}_{3}=-\mathrm{LP} 2$.

Step 2. Calculate the $\mathrm{Obj}_{1}, \mathrm{Obj}_{2}$, and $\mathrm{Obj}_{3}$ of all population chromosomes, and then rank them from small to large.

Step 3. After sorting chromosomes, the individual fitness of the three objectives is calculated and the overall fitness of each chromosome is calculated by sorting matrix [31].

$$
\begin{aligned}
& E_{j}(i)= \begin{cases}\left(N-R_{j}(i)\right)^{2} & R_{j}(i)>1 \\
k N^{2} & R_{j}(i)=1\end{cases} \\
& E(i)=E_{1}(i)+E_{2}(i)+E_{3}(i),
\end{aligned}
$$

where $R_{j}(i)$ is the ranking number of the $j$ th objective function for chromosome $i$, and $j=1,2,3, E_{j}(i)$ is the fitness value of chromosome $i$ in objective $j, E(i)$ is the overall fitness of chromosome $i, N$ is the population size, and $k \epsilon$ $(1,2)$ is a constant to increase the fitness value for the optimal individual. 


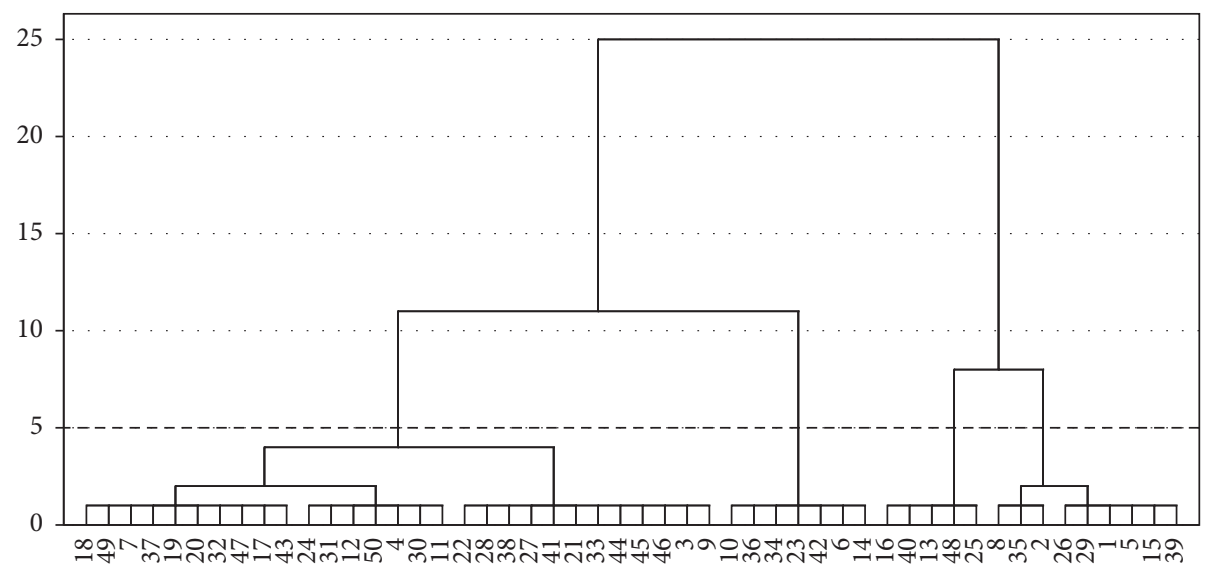

FIGURE 3: Results of the hierarchical clustering.

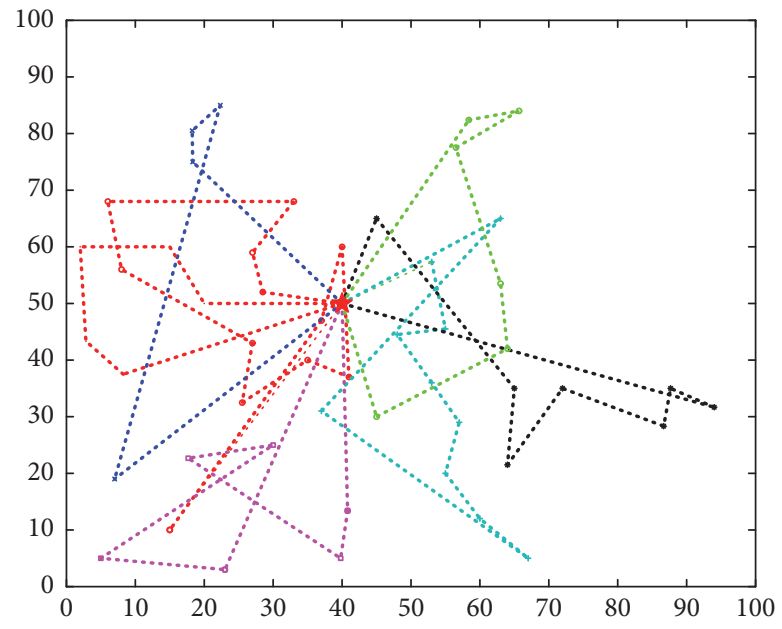

FIgURE 4: Optimal vehicle routes results by PB-GA.

\section{Numerical Experiments}

In order to validate the mathematical model and heuristic algorithm, numerical experiments are designed with a randomly generated 50-point example. Afterwards, the computational results are shown and the proposed method is compared. Furthermore, sensitivity analysis of the shelf life of perishable products is presented to explore the applicability of this paper.

5.1. Data Description. Fifty customer nodes are randomly generated on the two-dimensional plane with a range between $(0 \mathrm{~km}, 0 \mathrm{~km})$ and $(100 \mathrm{~km}, 100 \mathrm{~km})$. The depot is located at $(40 \mathrm{~km}, 50 \mathrm{~km})$. For simplicity, we set the lower and upper bound of fuzzy time window $e e_{i}=0, e l_{i}=10$, and service time $\mathrm{ST}_{i}=5 \mathrm{~min}$. More detailed customer data are shown in Table 1 , such as $(X, Y)$, expected arrival time window $\left[e_{i}, l_{i}\right]$, and customer demand $q_{i}$. Another parameter setting is shown in Table 2.
5.2. Results of Hierarchical Clustering for Customer Service Priority. In this section, we use the hierarchical clustering method to divide 50 customer priorities into several groups. Figure 3 shows the clustering results. Noticeably, when the reference value equals 5, all 50 customers are divided into four clusters.

In terms of clustering results, we can calculate average priority value for each cluster $\left(\operatorname{AVG} P^{\prime}(i)\right)$ in Table 3 . As we illustrate in Section 3.2, a small value of $P^{\prime}(i)$ corresponds to a high service priority for customer $i$. Therefore if a cluster has a small value of average priority, it comes to a high service priority for this cluster. As average priority value of cluster 1 is smallest among the other clusters, which present the highest priority, we set it as $P_{i}=1$. By that analogy, four other clusters are assigned to four different service priority levels. The service priority level for customer $i$ can be expressed as $P_{i}=\{1,2,3,4\}$, where $P_{i}=1$ represents the highest priority and $P_{i}=4$ represents the lowest one. In this way, customers will be served from high to low priority.

5.3. Experimental Results of Vehicle Scheduling. Numerical experiments are implemented with MATLAB R2011b. We set GA parameters as follows: population size $N=200$, crossover rate $P_{c}=0.8$, mutation rate $P_{m}=0.01$, and maximum iterations gen $=500$. Ten times of experiments by PB-GA and T-GA are carried out, respectively. The optimal vehicle routes for each algorithm are as follows.

(1) Optimal Vehicle Routes by PB-GA. Table 4 and Figure 4 show the optimal vehicle schedule by PB-GA. The result indicates that the delivery plans by PB-GA are directly affected by the priority constraint; namely, the service sequence order established in each subroute is from high priority to low. In this situation, the number of vehicles is 7 , customer satisfaction LP1 is $80.12 \%$, and the total cost LP2 is 1294.26 .

(2) Optimal Vehicle Routes by T-GA. Table 5 and Figure 5 describe the optimal vehicle schedule by T-GA where the number of vehicles is the same with that by PB-GA, but customers are not served as priority order. In this case, due 
TABLE 1: Details of customer node data with 50 customers.

\begin{tabular}{|c|c|c|c|}
\hline Customer & $(X, Y)$ & {$\left[e_{i}, l_{i}\right] / \mathrm{h}$} & $q_{i} / \mathrm{kg}$ \\
\hline 0 & $(40,50)$ & {$[0,10]$} & 0 \\
\hline 1 & $(22,85)$ & {$[0,1.2]$} & 50 \\
\hline 2 & $(18,75)$ & {$[0,1.3]$} & 70 \\
\hline 3 & $(26,33)$ & {$[0,1.4]$} & 18 \\
\hline 4 & $(45,65)$ & {$[1.1,1.6]$} & 9 \\
\hline 5 & $(18,23)$ & {$[0,2]$} & 49 \\
\hline 6 & $(41,37)$ & {$[2.2,2.7]$} & 16 \\
\hline 7 & $(5,5)$ & {$[1.2,1.7]$} & 16 \\
\hline 8 & $(18,81)$ & {$[0,1.1]$} & 70 \\
\hline 9 & $(60,12)$ & {$[1.3,1.8]$} & 31 \\
\hline 10 & $(20,50)$ & {$[1.5,2]$} & 5 \\
\hline 11 & $(67,5)$ & {$[2.4,2.9]$} & 25 \\
\hline 12 & $(64,42)$ & {$[1.2,1.7]$} & 9 \\
\hline 13 & $(8,38)$ & {$[0,1.5]$} & 100 \\
\hline 14 & $(23,3)$ & {$[2.5,3]$} & 7 \\
\hline 15 & $(53,57)$ & {$[0,1.93]$} & 52 \\
\hline 16 & $(3,43)$ & {$[0,1.1]$} & 100 \\
\hline 17 & $(8,56)$ & {$[1.5,2]$} & 8 \\
\hline 18 & $(64,22)$ & {$[0,1.2]$} & 8 \\
\hline 19 & $(37,31)$ & {$[0.6,1.1]$} & 14 \\
\hline 20 & $(6,68)$ & {$[1.5,2]$} & 30 \\
\hline 21 & $(53,36)$ & {$[0,1.1]$} & 25 \\
\hline 22 & $(55,46)$ & {$[0,0.7]$} & 37 \\
\hline 23 & $(40,60)$ & {$[2.4,2.9]$} & 21 \\
\hline 24 & $(2,60)$ & {$[1.3,1.8]$} & 5 \\
\hline 25 & $(40,5)$ & {$[0,1.6]$} & 90 \\
\hline 26 & $(94,32)$ & {$[0,1.5]$} & 60 \\
\hline 27 & $(48,45)$ & {$[0,3.3]$} & 26 \\
\hline 28 & $(29,52)$ & {$[0,1.2]$} & 36 \\
\hline 29 & $(88,35)$ & {$[0,1.5]$} & 60 \\
\hline 30 & $(57,29)$ & {$[1.6,2.1]$} & 18 \\
\hline 31 & $(30,25)$ & {$[1.6,2.1]$} & 23 \\
\hline 32 & $(55,20)$ & {$[1.4,19]$} & 19 \\
\hline 33 & $(87,28)$ & {$[0,1.4]$} & 30 \\
\hline 34 & $(37,47)$ & {$[1.4,1.9]$} & 6 \\
\hline 35 & $(66,84)$ & {$[0,1.2]$} & 70 \\
\hline 36 & $(15,10)$ & {$[2.5,3]$} & 20 \\
\hline 37 & $(63,65)$ & {$[0.5,1]$} & 8 \\
\hline 38 & $(27,59)$ & {$[0,1.6]$} & 35 \\
\hline 39 & $(7,19)$ & {$[0,1.2]$} & 54 \\
\hline 40 & $(58,82)$ & {$[0,1.1]$} & 100 \\
\hline 41 & $(57,78)$ & {$[0,1.1]$} & 30 \\
\hline 42 & $(15,60)$ & {$[2.6,3.1]$} & 17 \\
\hline 43 & $(27,43)$ & {$[0.7,1.2]$} & 9 \\
\hline 44 & $(33,68)$ & {$[0,3.5]$} & 26 \\
\hline 45 & $(63,54)$ & {$[0,1.6]$} & 17 \\
\hline 46 & $(35,40)$ & {$[0.2,0.7]$} & 16 \\
\hline 47 & $(65,35)$ & {$[0.9,1.4]$} & 3 \\
\hline 48 & $(41,13)$ & {$[0,1.7]$} & 110 \\
\hline
\end{tabular}

TABLE 1: Continued.

\begin{tabular}{lccc}
\hline Customer & $(X, Y)$ & {$\left[e_{i}, l_{i}\right] / \mathrm{h}$} & $q_{i} / \mathrm{kg}$ \\
\hline 49 & $(45,30)$ & {$[0.9,1.4]$} & 17 \\
50 & $(72,35)$ & {$[1.9,2.4]$} & 30 \\
\hline
\end{tabular}

TABLE 2: Parameter setting of experiments.

\begin{tabular}{lc}
\hline Parameter & Value \\
\hline Transport cost per unit distance $(¥ / \mathrm{km})$ & $c_{0}=0.5$ \\
Fixed vehicle cost per trip $(¥)$ & $F_{c}=100$ \\
Service time for customer $i(\mathrm{~min})$ & $\mathrm{ST}_{i}=5$ \\
Long-distance travel time before arriving LDC $(\mathrm{h})$ & $\Delta t=12$ \\
Maximum capacity per vehicle $(\mathrm{kg})$ & $\mathrm{Q}=300$ \\
Average travel speed $(\mathrm{km} / \mathrm{h})$ & $v_{0}=40$ \\
Shelf life for perishable product $(\mathrm{h})$ & $T=40$ \\
\hline
\end{tabular}

to the loose of priority constraint, the customer satisfaction LP1 is $74.96 \%$ which is lower than that by PG-GA and the total cost LP2 drops to 1272.57 .

\subsection{Experimental Results Analysis}

5.4.1. Results Analysis 1: Comparison of Optimal Results. This paper conducts a further comparison of optimal gaps in subgoal LP1 (customer satisfaction) and subgoal LP2 (the total costs) between two algorithms. Components of each subgoal are shown in Table 6.

(1) Compared with T-GA, the optimal gap of PB-GA in subgoal LP1 is $6.89 \%$. In particular, the degrees of satisfaction with freshness and time window are effective, with the improvement of $3.29 \%$ and $12.27 \%$, respectively. Thus, it reveals that PB-GA can promote the freshness of perishable products as well as achieve better solutions to satisfy the expected arrival time for customers.

(2) In terms of subgoal LP2, PB-GA has higher total costs than T-GA. However, with an overall perspective of two subobjectives, PB-GA only costs $1.7 \%$ more and gets $6.89 \%$ improvement rate in customer satisfaction with the same number of vehicles. Obviously, this increased cost is acceptable for logistics service providers, whereas the customer satisfaction can be enhanced significantly in comparison with T-GA. Thus, PB-GA tends to achieve better solutions for terminal delivery of perishable products.

5.4.2. Results Analysis 2: Sensitivity Analysis of Shelf Life for Perishable Products. It is worth noting that perishable products are characterized as time-sensitive items. Their shelf life has a great influence on perishable products' quality and freshness. In this section, we make a sensitivity analysis of shelf life to explore two algorithms' effects on customer satisfaction and the total costs.

It is observed from Figure 6 that as shelf life of products becomes longer, both of the algorithms have stronger effect. That is because a longer shelf life will keep perishable products with higher quality of freshness; moreover, it can increase customer satisfaction. Additionally, it is evident from this figure that PB-GA gets better solutions in customer 
TABLE 3: Service priority for customer nodes.

\begin{tabular}{lcc}
\hline Cluster & Customer nodes & Average priority value for each cluster $\left(\right.$ AVG $\left.P^{\prime}(i)\right)$ \\
\hline 1 & $\{13,16,25,40,48\}$ & 0.060 \\
2 & $\{1,2,5,8,15,26,29,35,39\}$ & 0.288 \\
3 & $\{3,7,9,11,12,17,18,19,20,21,22,24,27,28,30$, & 0.550 \\
4 & $31,32,33,37,38,41,43,44,45,46,47,49,50\}$ & 0.827 \\
\hline
\end{tabular}

TABLE 4: Optimal vehicle schedule by PB-GA.

\begin{tabular}{|c|c|c|c|c|c|}
\hline \multirow{2}{*}{ Vehicle schedule } & \multirow{2}{*}{ Priority } & \multicolumn{2}{|c|}{ Customer satisfaction LP1/\% } & \multicolumn{2}{|c|}{ Total costs LP2/¥ } \\
\hline & & Freshness & Time window & Transport cost & Fixed cost \\
\hline $0-28-38-44-20-17-43-3-46-6-23-34-36-0$ & $2-2-2-2-2-2-2-2-1-1-1-1$ & 71.88 & 79.79 & 123.92 & 100.00 \\
\hline $0-15-22-27-21-30-32-9-11-19-37-0$ & $3-2-2-2-2-2-2-2-2-2$ & 73.48 & 95.75 & 95.21 & 100.00 \\
\hline $0-2-8-1-39-0$ & $3-3-3-3$ & 73.68 & 98.09 & 78.85 & 100.00 \\
\hline $0-26-29-33-50-18-47-4-0$ & $3-3-2-2-2-2-2$ & 72.65 & 89.57 & 84.07 & 100.00 \\
\hline $0-48-25-5-31-7-14-0$ & $4-4-3-2-2-1$ & 73.64 & 86.46 & 93.02 & 100.00 \\
\hline $0-40-35-41-45-12-49-0$ & $4-3-2-2-2-2$ & 73.83 & 95.75 & 67.72 & 100.00 \\
\hline $0-13-16-24-42-10-0$ & $4-4-2-1-1$ & 74.47 & 88.95 & 51.48 & 100.00 \\
\hline \multicolumn{2}{|c|}{ Number of vehicles $=7$} & \multicolumn{2}{|c|}{$\mathrm{LP} 1=80.12 \%$} & \multicolumn{2}{|c|}{$\mathrm{LP} 2=1294.26$} \\
\hline
\end{tabular}

TABLE 5: Optimal vehicle schedule by T-GA.

\begin{tabular}{|c|c|c|c|c|c|}
\hline \multirow{2}{*}{ Vehicle schedule } & \multirow{2}{*}{ Priority } & \multicolumn{2}{|c|}{ Customer satisfaction LP1/\% } & \multicolumn{2}{|c|}{ Total costs LP2/¥ } \\
\hline & & Freshness & Time window & Transport cost & Fixed cost \\
\hline $0-43-31-39-24-20-12-47-50-26-33-18-9-37-0$ & $1-2-3-1-2-1-1-2-3-2-1-2-1$ & 67.14 & 64.45 & 164.75 & 100.00 \\
\hline $0-3-36-7-14-11-32-6-46-38-2-44-41-0$ & $2-2-2-1-2-2-2-2-2-4-2-2$ & 66.58 & 67.55 & 140.66 & 1050.00 \\
\hline $0-27-22-21-49-30-29-45-35-4-23-0$ & $2-2-2-2-2-3-2-3-1-2$ & 71.50 & 85.76 & 93.19 & 100.00 \\
\hline $0-19-48-25-5-34-0$ & $2-4-4-3-1$ & 74.02 & 88.78 & 54.67 & 100.00 \\
\hline $0-13-16-17-42-10-0$ & $4-4-2-2-1$ & 74.43 & 91.86 & 47.50 & 100.00 \\
\hline $0-8-1-40-15-0$ & $3-4-4-3$ & 72.97 & 93.84 & 60.13 & 100.00 \\
\hline $0-28-0$ & 2 & 76.26 & 60.21 & 11.67 & 100.00 \\
\hline \multicolumn{2}{|c|}{ Number of vehicles $=7$} & \multicolumn{2}{|c|}{$\mathrm{LP} 1=74.96 \%$} & \multicolumn{2}{|c|}{ LP2 $=1272.57$} \\
\hline
\end{tabular}

TABLE 6: Comparison of the optimal results.

\begin{tabular}{|c|c|c|c|c|c|c|}
\hline & \multirow{2}{*}{ Number of vehicles } & \multirow{2}{*}{$\begin{array}{c}\mathrm{LP} 1 / \% \\
\text { Customer satisfaction }\end{array}$} & \multicolumn{2}{|c|}{ Satisfaction } & \multirow{2}{*}{$\begin{array}{c}\text { LP2/¥ } \\
\text { Total costs }\end{array}$} & \multirow{2}{*}{ Transport cost } \\
\hline & & & Freshness & Time window & & \\
\hline PB-GA & 7 & $80.12 \%$ & $73.38 \%$ & $90.24 \%$ & 1294.26 & 594.26 \\
\hline T-GA & 7 & $74.96 \%$ & $71.04 \%$ & $80.38 \%$ & 1272.57 & 572.57 \\
\hline Gap & $0 \%$ & $6.89 \%$ & $3.29 \%$ & $12.27 \%$ & $-1.70 \%$ & $-3.79 \%$ \\
\hline
\end{tabular}

satisfaction than T-GA within the shelf life interval of 20$55 \mathrm{~h}$. That is because customer service priority is set based on subgoal LP1, and PB-GA will make every effort to improve customer satisfaction. We can figure out that it is effective to solve the delivery problem of perishable products by PB-GA.

Figure 7 illustrates that the total cost by PB-GA is higher than that by T-GA. That is because PB-GA will have a concern about the priority of customer to guarantee the satisfaction, so it will make the cost a little more than T-GA. However, the two total costs are close.
Compared with T-GA, Table 7 shows the optimal gaps of subgoals LP1 and LP2 by PB-GA under different shelf lives. It shows that, between 20 and $40 \mathrm{~h}$, the optimal gap of satisfaction increases remarkably which is higher than the dropped cost gap. However, between 45 and $55 \mathrm{~h}$, the increased cost gap is higher than the improvement of customer satisfaction. That is because when shelf life turns to be quite long, it no longer has the feature of perishability. Under these circumstances, PB-GA does not fit the normal products with long shelf life and T-GA is better. But in this paper, 


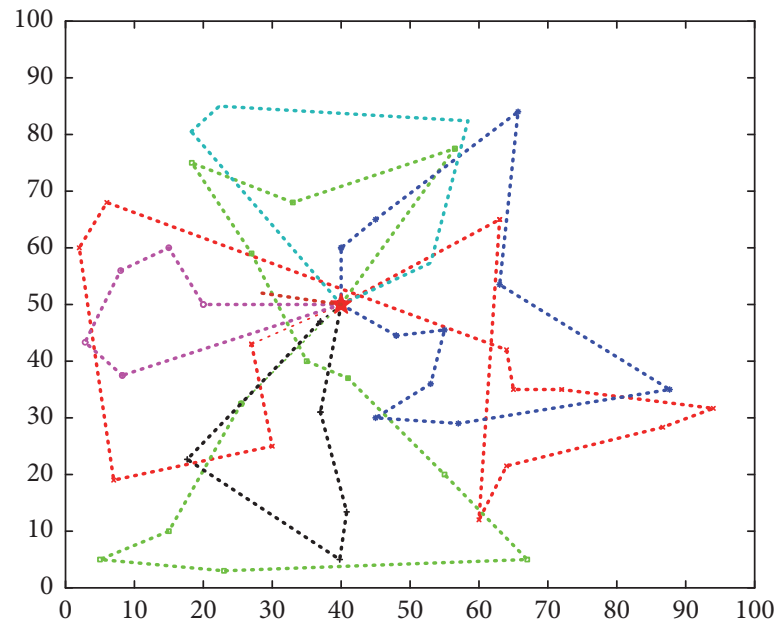

FIGURE 5: Optimal vehicle routes results by T-GA.

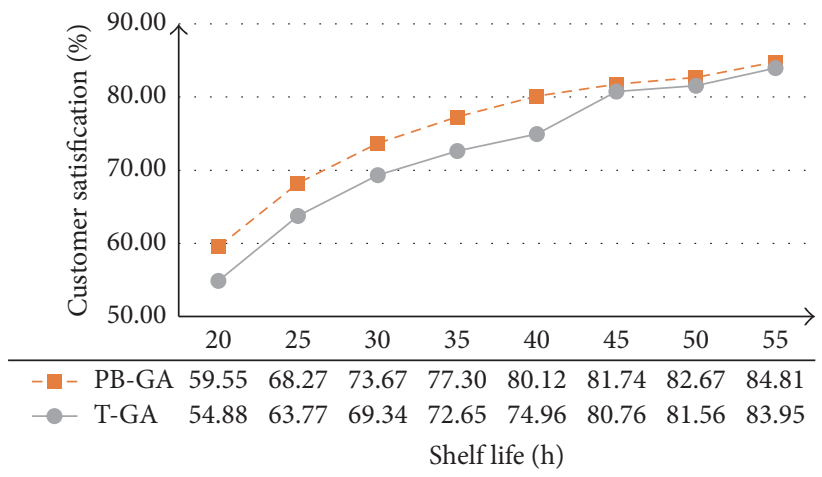

FIGURE 6: Results comparison of subgoal LP1.

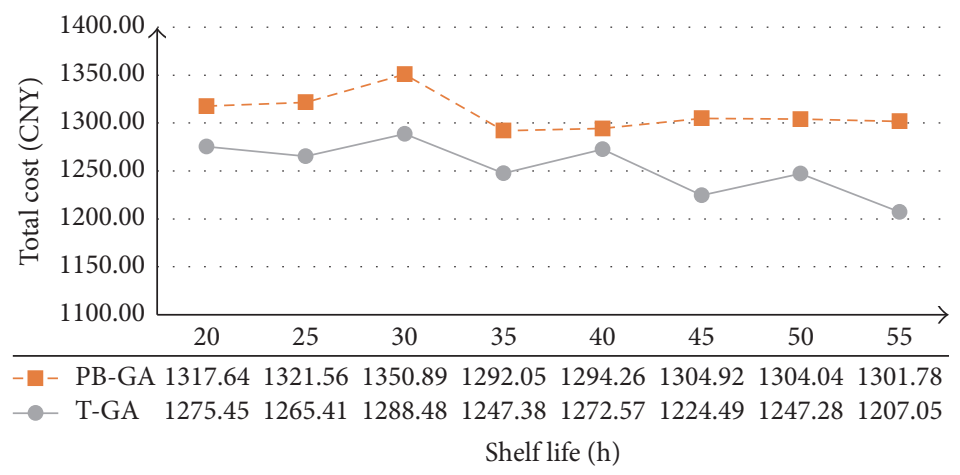

FIgURE 7: Results comparison of subgoal LP2.

TABLE 7: Optimal gaps of LP1 and LP2 by PB-GA.

\begin{tabular}{lccccccrc}
\hline Shelf life/h & 20 & 25 & 30 & 35 & 40 & 45 & 50 & \\
\hline Optimal gap/\% & & & & & & & \\
LP1 & 8.51 & 7.06 & 6.24 & 6.40 & 6.88 & 1.23 & 1.36 \\
LP2 & -3.31 & -4.41 & -4.84 & -3.58 & -1.70 & -6.57 & -4.55 & -7.85 \\
\hline
\end{tabular}


what we focus on are perishable products and the proposed algorithm still has a better effect on products within $45 \mathrm{~h}$ shelf life. So the applicability of PB-GA mainly focuses on perishable products with short shelf life.

\section{Conclusions}

This paper considers customer satisfaction in terminal delivery in order to deal with vehicle scheduling problem for perishable products. The main contributions of this paper include the following. (1) We qualify customer satisfaction from aspects of product freshness and time window. Based on priority evaluation function, we identify service priority for each customer as the service order in vehicle scheduling. (2) We construct a multiobjective vehicle scheduling optimization model for perishable products considering customer satisfaction. A priority-based genetic algorithm is designed to solve the problem. (3) Numerical experiments illustrate effectiveness of the proposed method which has improvements for customer satisfaction within a certain shelf life range. The sensitivity analysis reveals the adaptability in solving perishable products terminal delivery problem.

It is worth noting that some places need to be improved; for example, we did not consider multiple depots, multiple vehicle types, or multiple types of perishable products; however, these cases commonly exist in fresh products $\mathrm{B} 2 \mathrm{C}$ e-commerce environment. We will conduct further studies considering the above problems.

\section{Competing Interests}

The authors declare that they have no competing interests.

\section{Acknowledgments}

This work is supported by the National Natural Science Foundation of China by Grant (nos. 71471025, 71531002), the Natural Science Basic Research Project in Shaanxi Province (no. 2016JQ7005), China Ministry of Education Social Sciences and Humanities Research Youth Fund Project titled as "Delivery Optimization of Maturity-Based Fruit B2C Ecommerce-Taking Shaanxi Kiwifruit as the Example" (no. 16YJC630102), and Project funded by China Postdoctoral Science Foundation (no. 2016M600209).

\section{References}

[1] Chinese Internet Data Information Center, iiMedia Research: Research on China's Fresh Food Electronic Commerce Market in 2015, http://www.199it.com/archives/415016.html.

[2] J. Blackburn and G. Scudder, "Supply chain strategies for perishable products: the case of fresh produce," Production \& Operations Management, vol. 18, no. 2, pp. 129-137, 2009.

[3] A. A. Syntetos, "Perishable Inventory Systems," Journal of the Operational Research Society, vol. 65, no. 5, pp. 797-798, 2014.

[4] A. Osvald and L. Z. Stirn, "A vehicle routing algorithm for the distribution of fresh vegetables and similar perishable food," Journal of Food Engineering, vol. 85, no. 2, pp. 285-295, 2008.
[5] C.-I. Hsu, S.-F. Hung, and H.-C. Li, "Vehicle routing problem with time-windows for perishable food delivery," Journal of Food Engineering, vol. 80, no. 2, pp. 465-475, 2007.

[6] H.-K. Chen, C.-F. Hsueh, and M.-S. Chang, "Production scheduling and vehicle routing with time windows for perishable food products," Computers \& Operations Research, vol. 36, no. 7, pp. 2311-2319, 2009.

[7] O. Ahumada and J.-R. Villalobos, "A tactical model for planning the production and distribution of fresh produce," Agricultural Systems, vol. 112, no. 13, pp. 17-26, 2011.

[8] C. Yan, A. Banerjee, and L. Yang, "An integrated productiondistribution model for a deteriorating inventory item," International Journal of Production Economics, vol. 133, no. 1, pp. 228232, 2011.

[9] Y. Wang and L.-Y. Yu, "Optimization model of refrigerated food transportation," in Proceedings of the 9th International Conference on Service Systems and Service Management (ICSSSM '12), pp. 220-224, IEEE, Shanghai, China, July 2012.

[10] L. C. Coelho and G. Laporte, "Optimal joint replenishment, delivery and inventory management policies for perishable products," Computers and Operations Research, vol. 47, pp. 42$52,2014$.

[11] Z. Firoozi, N. Ismail, S. Ariafar et al., "Effects of integration on the cost reduction in distribution network design for perishable products," Mathematical Problems in Engineering, vol. 2014, Article ID 739741, 10 pages, 2014.

[12] J. Ruan and Y. Shi, "Monitoring and assessing fruit freshness in IOT-based e-commerce delivery using scenario analysis and interval number approaches," Information Sciences, vol. 373, pp. 557-570, 2016.

[13] A. Rong, R. Akkerman, and M. Grunow, "An optimization approach for managing fresh food quality throughout the supply chain," International Journal of Production Economics, vol. 131, no. 1, pp. 421-429, 2011.

[14] T. Jia, X. Li, N. Wang, and R. Li, "Integrated inventory routing problem with quality time windows and loading cost for deteriorating items under discrete time," Mathematical Problems in Engineering, vol. 2014, Article ID 537409, 14 pages, 2014.

[15] P. Amorim and B. Almada-Lobo, "The impact of food perishability issues in the vehicle routing problem," Computers \& Industrial Engineering, vol. 67, no. 1, pp. 223-233, 2014.

[16] Q. Cao, J.-P. Shao, and Y.-N. Sun, "Multi-objective for fresh agricultural product distribution path optimization based on improved genetic algorithm," Industrial Engineering and Management, vol. 20, no. 1, pp. 122-134, 2015.

[17] A. Stepanov and J. M. Smith, "Multi-objective evacuation routing in transportation networks," European Journal of Operational Research, vol. 198, no. 2, pp. 435-446, 2009.

[18] L. Sun, G. W. DePuy, and G. W. Evans, "Multi-objective optimization models for patient allocation during a pandemic influenza outbreak," Computers \& Operations Research, vol. 51, pp. 350-359, 2014.

[19] M. Najafi, K. Eshghi, and W. Dullaert, "A multi-objective robust optimization model for logistics planning in the earthquake response phase," Transportation Research Part E: Logistics and Transportation Review, vol. 49, no. 1, pp. 217-249, 2013.

[20] R. Abounacer, M. Rekik, and J. Renaud, "An exact solution approach for multi-objective location-transportation problem for disaster response," Computers and Operations Research, vol. 41, no. 1, pp. 83-93, 2014. 
[21] H. Wang, L. Du, and S. Ma, "Multi-objective open locationrouting model with split delivery for optimized relief distribution in post-earthquake," Transportation Research Part E: Logistics and Transportation Review, vol. 69, pp. 160-179, 2014.

[22] J. H. Ruan, X. P. Wang, F. T. S. Chan, and Y. Shi, "Optimizing the intermodal transportation of emergency medical supplies using balanced fuzzy clustering," International Journal of Production Research, vol. 54, no. 14, pp. 4368-4386, 2016.

[23] P. Amorim, H.-O. Günther, and B. Almada-Lobo, "Multiobjective integrated production and distribution planning of perishable products," International Journal of Production Economics, vol. 138, no. 1, pp. 89-101, 2012.

[24] N.-Z. Atashbar and A. Baboli, "A multi-objective routing problem for perishable items in a disaster relief," in Proceedings of the 44th International Conference on Computers and Industrial Engineering 2014 (CIE '44) and 9th International Symposium on Intelligent Manufacturing and Service Systems 2014 (IMSS '14), October 2014.

[25] M. Bortolini, M. Faccio, E. Ferrari, M. Gamberi, and F. Pilati, "Fresh food sustainable distribution: cost, delivery time and carbon footprint three-objective optimization," Journal of Food Engineering, vol. 174, no. 85, pp. 56-67, 2015.

[26] M. Rahimi, A. Baboli, and Y. Rekik, "Sustainable inventory routing problem for perishable products by considering reverse logistic," IFAC-PapersOnLine, vol. 49, no. 12, pp. 949-954, 2016.

[27] X.-P. Wang, M. Wang, J.-H. Ruan, and H. Zhan, "The multiobjective optimization for perishable food distribution route considering temporal-spatial distance," Procedia Computer Science, vol. 96, pp. 1211-1220, 2016.

[28] L. Lin, S.-P. Yang, and B. Dan, "Three-level supply chain coordination of fresh agricultural products with time constraints," Chinese Journal of Management Science, vol. 19, no. 3, pp. 55-62, 2011.

[29] X.-P. Wang, K. Zhang, and X.-P. Hu, "Research of vehicle routing problem based on fuzzy time windows," Journal of Industrial Engineering and Engineering Management, vol. 23, no. 3, pp. 148-154, 2011.

[30] S. C. Johnson, "Hierarchical clustering schemes," Psychometrika, vol. 32, no. 3, pp. 241-254, 1967.

[31] X.-P. Wang, J.-H. Ruan, K. Zhang et al., "Study on combinational disruption management for vehicle routing problem with fuzzy time windows," Journal of Management Sciences in China, vol. 14, no. 6, pp. 2-15, 2011. 


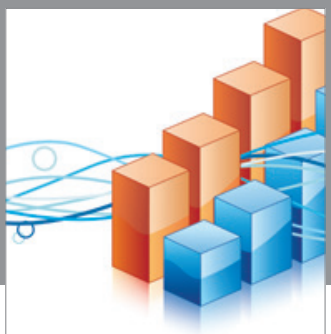

Advances in

Operations Research

vatem alat4

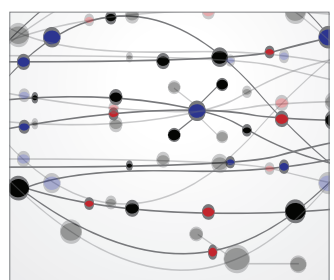

\section{The Scientific} World Journal
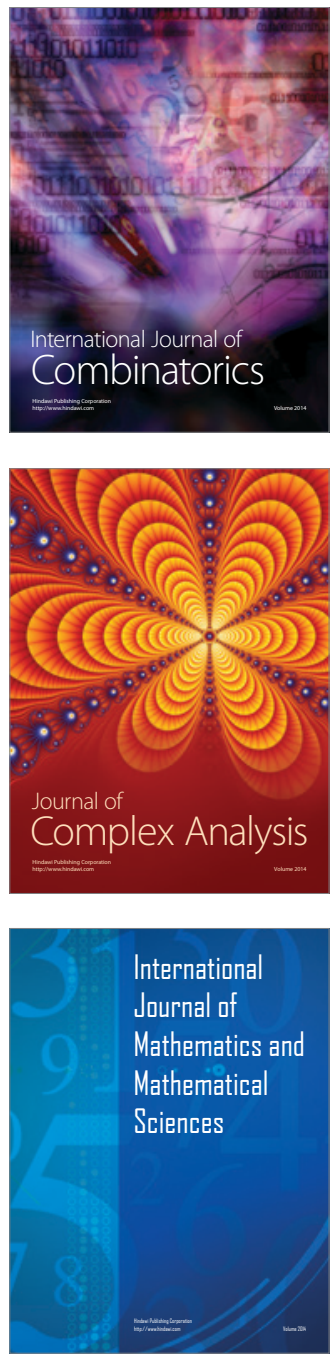
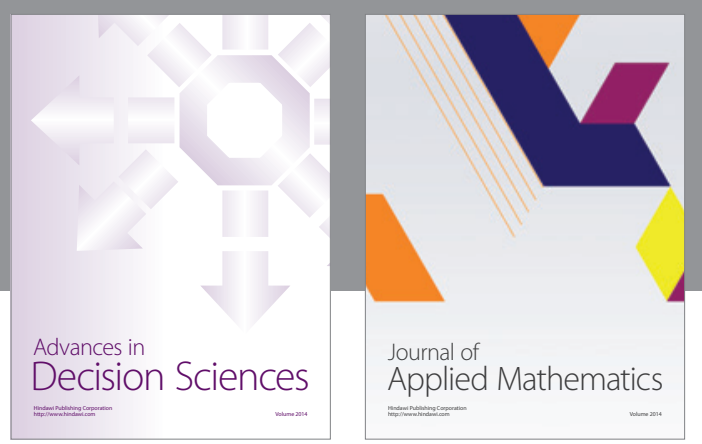

Algebra

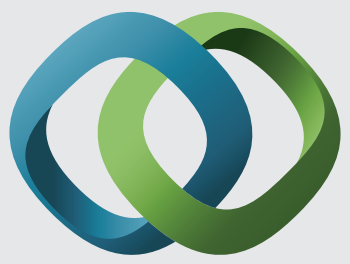

\section{Hindawi}

Submit your manuscripts at

https://www.hindawi.com
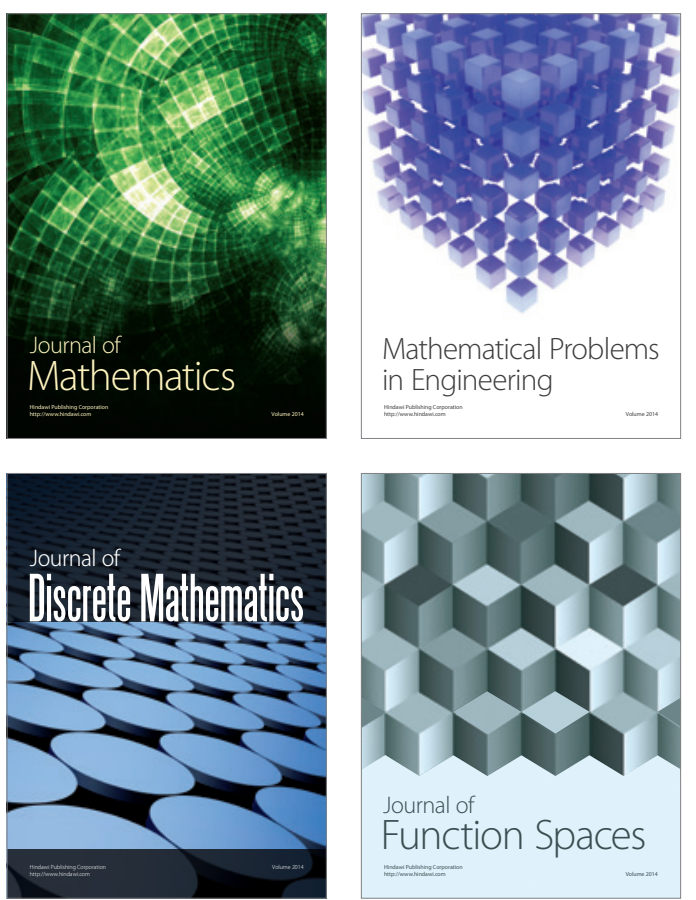

Mathematical Problems in Engineering
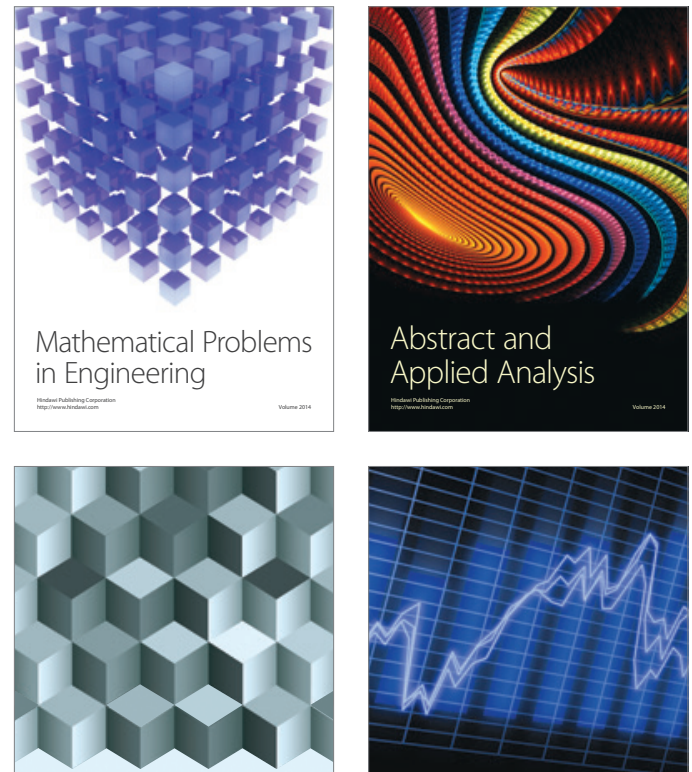

Journal of

Function Spaces

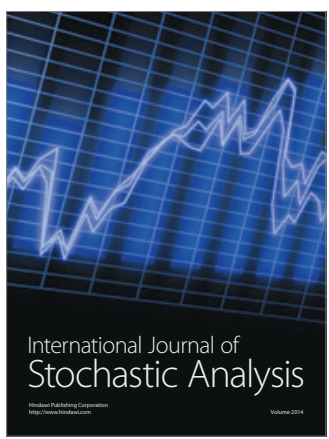

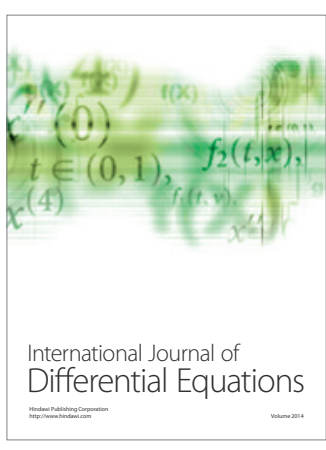
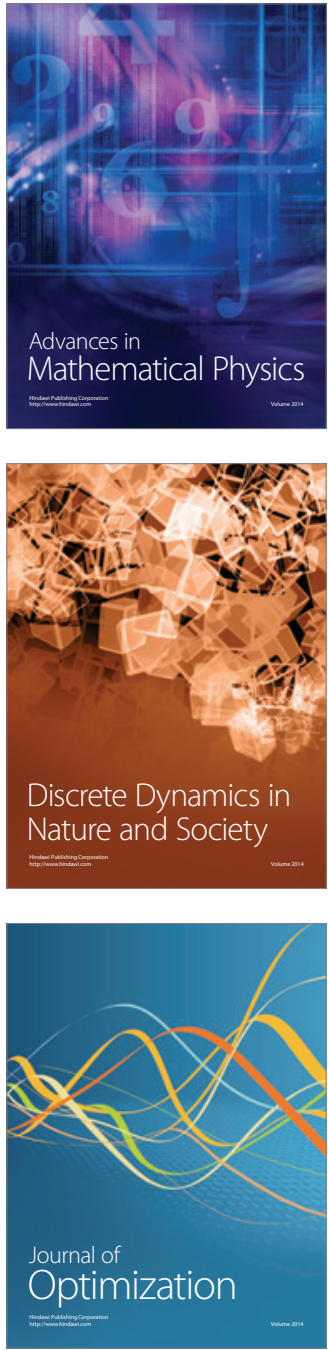